\title{
The Prospective Studies of Atherosclerosis (Proof-ATHERO) consortium: Design and rationale
}

Writing committee: Lena Tschiderer ${ }^{1 \S}$, Lisa Seekircher ${ }^{1 \S}$, Gerhard Klingenschmid ${ }^{1}$, Raffaele Izzo $^{2}$, Damiano Baldassarre ${ }^{3,4}$, Bernhard Iglseder ${ }^{5,6}$, Laura Calabresi ${ }^{7}$, Jing Liu ${ }^{8}$, Jackie F. Price $^{9}$, Jang-Ho Bae ${ }^{10,11}$, Frank P. Brouwers ${ }^{12}$, Eric de Groot $^{13}$, Caroline Schmidt ${ }^{14}$, Göran Bergström ${ }^{15,16}$, Gülay Aşçi ${ }^{17}$, Paolo Gresele ${ }^{18}$, Shuhei Okazaki ${ }^{19}$, Kostas Kapellas ${ }^{20}$, Manuel F. Landecho $^{21}$, Naveed Sattar ${ }^{22}$, Stefan Agewall ${ }^{23}$, Zhi-Yong Zou ${ }^{24}$, Christopher D. Byrne ${ }^{25}$, Prabath W. B. Nanayakkara ${ }^{26}$, Aikaterini Papagianni ${ }^{27}$, Miles D. Witham ${ }^{28}$, Enrique Bernal ${ }^{29}$, Robert Ekart $^{30}$, Michiel A. van Agtmael ${ }^{31}$, Mario F. Neves ${ }^{32}$, Eiichi Sato ${ }^{33}$, Marat Ezhov ${ }^{34}$, Matthew Walters ${ }^{35}$, Michael H. Olsen ${ }^{36}$, Radojica Stolić ${ }^{37}$, Dorota A. Zozulińska-Ziółkiewicz ${ }^{38}$, Markolf Hanefeld ${ }^{39}$, Daniel Staub ${ }^{40}$, Michiaki Nagai ${ }^{41}$, Pythia T. Nieuwkerk ${ }^{42}$, Menno V. Huisman $^{43}$, Akihiko Kato ${ }^{44}$, Hirokazu Honda ${ }^{45}$, Grace Parraga ${ }^{46}$, Dianna Magliano ${ }^{47}$, Rafael Gabriel $^{48}$, Tatjana Rundek ${ }^{49}$, Mark A. Espeland ${ }^{50}$, Stefan Kiechl ${ }^{1,51}$, Johann Willeit ${ }^{1}$, Lars Lind $^{52}$, Jean Philippe Empana ${ }^{53}$, Eva Lonn ${ }^{54,55}$, Tomi-Pekka Tuomainen ${ }^{56}$, Alberico Catapano $^{7,57}$, Kuo-Liong Chien ${ }^{58}$, Dirk Sander ${ }^{59,60}$, Maryam Kavousi ${ }^{61}$, Joline W. J. Beulens ${ }^{62}$, Michiel L. Bots ${ }^{63}$, Michael J. Sweeting ${ }^{64,65}$, Matthias W. Lorenz ${ }^{66}$, and Peter Willeit ${ }^{1,65 *}$ on behalf of the Proof-ATHERO Study Group

$\S^{\S}$ Denotes equal contribution.

The members of the collaborators committee are listed at end of the paper.

${ }^{1}$ Department of Neurology, Medical University of Innsbruck, Innsbruck, Austria; ${ }^{2}$ Department of Advanced Biochemical Sciences, Federico II University, Naples, Italy; ${ }^{3}$ Department of Medical Biotechnology and Translational Medicine, University of Milan, Milan, Italy; ${ }^{4}$ Centro Cardiologico Monzino IRCCS, Milan, Italy; ${ }^{5}$ Department of Geriatric Medicine, Gemeinnützige Salzburger Landeskliniken Betriebsgesellschaft GmbH Christian-DopplerKlinik, Salzburg, Austria; ${ }^{6}$ Department of Geriatric Medicine, Paracelsus Medical University, Salzburg, Austria; ${ }^{7}$ Department of Pharmacological and Biomolecular Sciences, University of Milan, Milan, Italy; ${ }^{8}$ Department of Epidemiology, Beijing Anzhen Hospital, Capital Medical University, Beijing, China; ${ }^{9}$ Usher Institute, University of Edinburgh, Edinburgh, UK; ${ }^{10} \mathrm{Heart}$ Center, Konyang University Hospital, Daejeon, Korea; ${ }^{11}$ Department of Cardiology, Konyang University College of Medicine, Daejeon, Korea; ${ }^{12}$ Department of Cardiology, Haga Teaching Hospital, the Hague, the Netherlands; ${ }^{13}$ Imagelabonline \& Cardiovascular, Eindhoven and Lunteren, the Netherlands; ${ }^{14}$ Wallenberg Laboratory for Cardiovascular Research, University of Gothenburg, Gothenburg, Sweden; ${ }^{15}$ Department of Molecular and Clinical Medicine, Institute of Medicine, Sahlgrenska Academy, University of Gothenburg, Gothenburg, Sweden; ${ }^{16}$ Department of Clinical Physiology, Sahlgrenska University Hospital, Region Västra Götaland, Gothenburg, Sweden; ${ }^{17}$ Nephrology Department, Ege University School of Medicine, Bornova-Izmir, Turkey; ${ }^{18}$ Division of Internal and Cardiovascular Medicine, Department of Medicine, University of Perugia, Perugia, Italy; ${ }^{19}$ Department of Neurology, Osaka University Graduate School of Medicine, Osaka, Japan; ${ }^{20}$ Australian Research Centre for Population Oral Health, University of Adelaide, Adelaide, SA, Australia; ${ }^{21}$ Department of Internal Medicine, University Clinic of Navarra, Navarra, Spain; ${ }^{22} \mathrm{BHF}$ Glasgow Cardiovascular Research Centre, University of Glasgow, Glasgow, UK; ${ }^{23}$ Oslo University Hospital Ullevål and Institute of Clinical Sciences, University of Oslo, Oslo, Norway; ${ }^{24}$ Institute of Child and Adolescent Health, School of Public Health, Peking University, Beijing, China; ${ }^{25}$ Human Development and Health Academic Unit, Faculty of Medicine, The Institute of Developmental Sciences, University of Southampton - Southampton General Hospital, Southampton, UK; ${ }^{26}$ Department of Clinical Neurophysiology, Amsterdam UMC, Amsterdam, the Netherlands; ${ }^{27}$ University Department of Nephrology, Hippokration General Hospital, 
Thessaloniki, Greece; ${ }^{28}$ AGE Research Group, NIHR Newcastle Biomedical Research Centre, Newcastle University and Newcastle-upon-Tyne Hospitals Trust, Newcastle, UK; ${ }^{29}$ Infectious Diseases Unit, Reina Sofia Hospital, Murcia, Spain; ${ }^{30}$ Department of Dialysis, University Medical Centre Maribor, Maribor, Slovenia; ${ }^{31}$ Department of Internal Medicine Amsterdam UMC, Vrije Universiteit, Amsterdam, the Netherlands; ${ }^{32}$ Department of Clinical Medicine, State University of Rio de Janeiro, Rio de Janeiro, Brazil; ${ }^{33}$ Division of Nephrology, Shinmatsudo Central General Hospital, Chiba, Japan; ${ }^{34}$ Laboratory of Lipid Disorders, National Medical Research Center of Cardiology, Moscow, Russia; ${ }^{35}$ School of Medicine, Dentistry and Nursing, University of Glasgow, Glasgow, UK; ${ }^{36}$ Department of Internal Medicine, Holbaek Hospital, University of Southern Denmark, Odense, Denmark; ${ }^{37}$ Department of Internal Medicine, Faculty of Medical Sciences, University of Kragujevac, Kragujevac, Serbia; ${ }^{38}$ Department of Internal Medicine and Diabetology, Poznan University of Medical Sciences, Poznan, Poland; ${ }^{39}$ Center for Clinical Studies, Technical University Dresden, Dresden, Germany; ${ }^{40}$ Department of Angiology, University Hospital Basel, Basel, Switzerland; ${ }^{41}$ Department of Internal Medicine, General Medicine and Cardiology, Hiroshima City Asa Hospital, Hiroshima, Japan; ${ }^{42}$ Department of Medical Psychology, Amsterdam UMC- Location AMC, Amsterdam, the Netherlands; ${ }^{43}$ Department of Thrombosis and Hemostasis, Leiden University Medical Center, Leiden, the Netherlands; ${ }^{44}$ Blood Purification Unit, Hamamatsu University Hospital, Hamamatsu, Japan; ${ }^{45}$ Division of Nephrology, Department of Medicine, Showa University School of Medicine, Tokyo, Japan; ${ }^{46}$ Department of Medical Biophysics, Western University, London, Canada; ${ }^{47}$ Department of Epidemiology and Preventive Medicine, Monash University, Alfred Hospital, Melbourne, Australia; ${ }^{48}$ National School of Public Health, Instituto de Salud Carlos III, Madrid, Spain; ${ }^{49}$ Department of Neurology, University of Miami Miller School of Medicine, Miami, USA; ${ }^{50}$ Department of Biostatistical Sciences, Wake Forest School of Medicine, Winston-Salem, NC, USA; ${ }^{51}$ VASCage GmbH, Research Centre on Vascular Ageing and Stroke, Innsbruck, Austria; ${ }^{52}$ Department of Medicine, Uppsala University, Uppsala, Sweden; ${ }^{53}$ Paris Cardiovascular Research Centre (PARCC), University Paris Descartes, Paris, France; ${ }^{54}$ Department of Medicine and Population Health Research Institute, McMaster University, Hamilton, Ontario, Canada; ${ }^{55}$ Hamilton General Hospital, Hamilton, Ontario, Canada; ${ }^{56}$ Institute of Public Health and Clinical Nutrition, University of Eastern Finland, Kuopio Campus, Kuopio, Finland; ${ }^{57}$ IRCCS Multimedica, Milan, Italy; ${ }^{58}$ Institute of Epidemiology and Preventive Medicine, National Taiwan University, Taipei, Taiwan; ${ }^{59}$ Department of Neurology, Benedictus Hospital Tutzing \& Feldafing, Feldafing, Germany; ${ }^{60}$ Department of Neurology, Technische Universität München, Munich, Germany; ${ }^{61}$ Department of Epidemiology, Erasmus University Medical Center, Rotterdam, the Netherlands; ${ }^{62}$ Department of Epidemiology \& Biostatistics, Amsterdam UMC- Location Vumc, Amsterdam, the Netherlands; ${ }^{63}$ Julius Center for Health Sciences and Primary Care, University Medical Center Utrecht, Utrecht, the Netherlands; ${ }^{64}$ Department of Health Sciences, University of Leicester, Leicester, UK; ${ }^{65}$ Department of Public Health and Primary Care, University of Cambridge, Cambridge, UK; ${ }^{66}$ Department of Neurology, Goethe University, Frankfurt am Main, Germany

Short Title: Design and rationale of the Proof-ATHERO consortium

\section{*Corresponding author:}

Peter Willeit, MD MPhil PhD, Department of Neurology, Medical University of Innsbruck, Anichstraße 35, 6020 Innsbruck, Austria, Tel: +43 512 504-83493, Fax: +43 50 504-23852, Email: peter.willeit@i-med.ac.at

$\mathbf{2}$ tables, $\mathbf{3}$ figures, $\mathbf{3 8 8 4}$ words 


\begin{abstract}
Atherosclerosis - the pathophysiological mechanism shared by most cardiovascular diseases can be directly or indirectly assessed by a variety of clinical tests including measurement of carotid intima-media thickness, carotid plaque, ankle-brachial index, pulse wave velocity, and coronary artery calcium. The Prospective Studies of Atherosclerosis (Proof-ATHERO) Consortium (https://clinicalepi.i-med.ac.at/research/proof-athero/) collates de-identified individual-participant data of studies with information on atherosclerosis measures, risk factors for cardiovascular disease, and incidence of cardiovascular diseases. It currently comprises 74 studies that involve 106,846 participants from 25 countries and over 40 cities. 21 studies recruited participants from the general population $(n=67,784), 16$ from high-risk populations $(n=22,677)$, and 37 as part of clinical trials $(n=16,385)$. Baseline years of contributing studies range from April 1980 to July 2014; the latest follow-up was until June 2019. Mean age at baseline was 59 (standard deviation: 10) years and 50\% were female. Over a total of 830,619 person-years of follow-up, 17,270 incident cardiovascular events (including coronary heart disease and stroke) and 13,270 deaths were recorded, corresponding to cumulative incidences of $2.1 \%$ and $1.6 \%$ per annum. The consortium is coordinated by the Clinical Epidemiology Team at the Medical University of Innsbruck, Austria. Contributing studies undergo a detailed data cleaning and harmonisation procedure before being incorporated in the Proof-ATHERO central database. Statistical analyses are being conducted according to pre-defined analysis plans and use established methods for individual-participant data meta-analysis. Capitalising on its large sample size, the multi-institutional collaborative Proof-ATHERO consortium aims to better characterise, understand, and predict the development of atherosclerosis and its clinical consequences.
\end{abstract}

Keywords: Prospective studies · Consortium - Individual-participant data $\cdot$ Atherosclerosis · Repeat measurements $\cdot$ Cardiovascular disease 


\section{Introduction}

Cardiovascular diseases (CVD) are the most common cause of death and disability worldwide. According to recent estimates from the Global Burden of Disease Study, about 18 million people die of CVD in a year, which account for over $30 \%$ of all global deaths [1]. The pathophysiological mechanism shared by many CVD is atherosclerosis, a gradual and progressive hardening and narrowing of the arteries over the course of life. Initial atherosclerotic alterations can be found as early as in young adulthood [2, 3] and involve endothelial dysfunction, inflammation, and deposition of fat [4]. Advanced atherosclerotic lesions are characterised by formation of atherosclerotic plaque that can destabilise, rupture or fissure, and can ultimately lead to acute vessel occlusion or formation of a local thrombus with dislocation into distal arteries and thereby clinical sequelae [4].

Clinical and subclinical atherosclerosis can be directly or indirectly assessed using a range of different clinical tests which are simple, safe, and non-invasive, and therefore amenable for use in large-scale studies (Fig. 1). One of the imaging techniques for atherosclerosis most frequently used is the assessment of carotid intima-media thickness (cIMT). Using B-mode high-resolution ultrasound, the distance between the adventitia-media interface and the intima-lumen interface of the carotid arterial wall is quantified. Spatial resolution of this imaging technique is approximately $50 \mu \mathrm{m}$ axially and $200 \mu \mathrm{m}$ laterally. Ultrasound-based cIMT is considered as a marker of the early stage of atherosclerosis. It is related to unfavourable levels of traditional cardiovascular risk factors $[5,6]$ and has been shown to be in good accordance with "true" cIMT determined in histological studies [7]. Furthermore, increased cIMT has been associated with increased risk of cardiovascular events $[8,9]$.

Other scalable and commonly available measures to ascertain vessel wall pathology and dysfunction include carotid plaque [10,11], ankle-brachial index [12], pulse wave velocity [13], and the coronary artery calcium score [14-16] (Fig. 1). As reviewed recently [17], these measures have several strengths and weaknesses. cIMT, carotid plaque, ankle-brachial index, and pulse wave velocity are non-invasive and cost-effective markers, which are therefore relatively easy to implement in large clinical studies. However, disadvantages include measurement error and lack of standardisation in measurement protocols for cIMT, specificity of ankle-brachial index [12], and the error associated with the measurement of travelled distance for pulse wave velocity [18]. The coronary artery calcium score directly quantifies presence of calcification in coronary arteries [19]. In contrast to the other mentioned markers, coronary 
artery calcification is assessed with computed tomography, which is more costly and exposes the study participant to radiation, thereby limiting large-scale assessments.

According to the 2019 European Society of Cardiology Guidelines for the diagnosis and management of chronic coronary syndromes, atherosclerotic plaque detection by carotid artery ultrasound, assessment of coronary artery calcium score with computed tomography, and measurement of the ankle-brachial index may be considered as risk modifiers in cardiovascular risk assessment in asymptomatic subjects [19]. Because atherosclerosis typically develops over a long period of time and only causes symptoms at an advanced stage, these measures are important tools in clinical practice to quantify atherosclerosis burden and might help inform treatment decisions.

The Prospective Studies of Atherosclerosis (Proof-ATHERO) consortium is an international consortium that brings together individual-participant data from prospective cohorts with detailed information on atherosclerosis, covariates, and incidence of CVD outcomes. The present report provides a description of broad aims of the Proof-ATHERO consortium and the principal methodology involved in collating, harmonising, and analysing study data.

\section{Design}

\section{Objectives}

Capitalising on its large sample size and the comprehensive information available, the overarching aims of the Proof-ATHERO consortium are to: (i) better characterise the natural history, communalities, and differences of different atherosclerosis measures; (ii) to provide novel insight into the determinants of atherosclerosis development and progression; and (iii) to investigate clinical consequences of atherosclerosis. In contrast to prior reports in individual studies, the large-scale data of Proof-ATHERO enables the study team to conduct powerdemanding analyses, including (i) characterisation of atherosclerosis trajectories over time; (ii) determination of the shapes of associations (e.g. linear vs. curvilinear vs. threshold effects); (iii) study of potential effect modifiers (e.g. age, sex, medication, or different lifestyle factors such as smoking habit); (iv) direct comparisons of the added predictive value of different atherosclerosis measures over and beyond assessment of conventional risk factors; and (v) reliable evaluation of atherosclerosis measures as surrogate markers for clinically manifest 
CVD endpoints. Overall, Proof-ATHERO aims to analyse world-wide available data to deliver results based on the highest scientific evidence.

\section{$\underline{\text { Inclusion criteria }}$}

Prospective cohorts are eligible for inclusion in the Proof-ATHERO consortium if they were observational studies or clinical trials that: (i) have assessed one or more atherosclerosis measures (i.e. cIMT, carotid plaque, ankle-brachial index, pulse wave velocity, and coronary artery calcium) repeatedly (i.e. at two or more time points); (ii) have ascertained comprehensive information on CVD risk factors (e.g. lifestyle, blood-based markers, history of disease, and medication intake); and (iii) have recorded incident CVD outcomes using well-defined criteria.

A crucial foundation for the Proof-ATHERO consortium was provided by the PROGIMT project [20]. This initiative led by Matthias Lorenz at the Goethe University at Frankfurt am Main had collated and analysed individual-participant data on the progression of cIMT and, for instance, yielded milestone publications on the association of cIMT progression with future CVD risk in the general population [8], in people with type-2 diabetes [21], and in people at high cardiovascular risk [22]. When the PROG-IMT project was completed in 2017, a majority of contributing studies ( $83 \%$ ) decided to continue the fruitful collaboration as part of the ProofATHERO consortium and to jointly investigate scientific questions which go beyond the initial aims of the PROG-IMT project. The commitment by these studies gave a unique head-start to the Proof-ATHERO consortium and enabled efficient data accrual at the beginning of the initiative.

Identification and incorporation of new eligible studies is ongoing and we invite researchers to contact the coordinating centre if they wish to contribute to the Proof-ATHERO consortium.

\section{$\underline{\text { Atherosclerosis measures }}$}

Data have been sought from investigators on carotid ultrasound parameters, ankle-brachial index, pulse wave velocity, and coronary artery calcium at baseline and any subsequent reexaminations during follow-up. Atherosclerosis measures assessed by the individual studies are summarised in Table 1. Parameters based on carotid ultrasound are being collected systematically on up to twelve sites (common carotid artery, carotid bifurcation, and internal carotid artery; left and right side; near and far wall) and include cIMT, vessel diameter, presence of plaques (yes vs. no), number of plaques, plaque thickness (height in mm), plaque area in a 
longitudinal view (in $\mathrm{mm}^{2}$ ), and plaque morphology according to the Gray-Weale classification [23]. The methodologies which studies used to cIMT and carotid plaque are summarised in Table S2 and Table S3, respectively.

\section{Participant characteristics at the baseline and follow-up surveys}

Data on participant characteristics at baseline and follow-up surveys have been sought from investigators on age, sex, ethnicity, socio-economic status, smoking, systolic and diastolic blood pressure, body-mass index, lipid markers (e.g. total cholesterol, high- and low-density lipoprotein cholesterol, triglycerides), markers of inflammation (e.g. C-reactive protein, fibrinogen, leukocyte count), markers of dysglycaemia (e.g. fasting glucose, glycated haemoglobin), use of medication (e.g. antihypertensive, antidiabetic, lipid-lowering medication), and pre-existing diseases (e.g. coronary heart disease, stroke, diabetes, or hypertension). Furthermore, in clinical trials, information on the type of interventions (and dosages, if appropriate) and on adherence to allocated regimens have been collated.

\section{$\underline{\text { Incident disease outcomes }}$}

Data on incident disease outcomes have been collated predominantly on fatal and non-fatal CVD events, including myocardial infarction, angina pectoris, and subtypes of stroke. Details on the ascertainment of prevalent and incident CVD are provided in Table S4. Studies assessed prevalent CVD at study baseline using self-report only or supplemented by objective criteria.

The vast majority of the studies used objective criteria rather than self-report only for assessing incident coronary heart disease (93\%) and incident stroke $(90 \%)$. Outcomes were classified according to International Classification of Diseases-10 coding (e.g. I20-I25 for coronary heart disease and I61-I69 for stroke) or to study-specific coding systems. In addition, information on cause-specific death has been sought. In 15 studies, cause of death was ascertained based on the death certificate; 44 studies supplemented the death certificate with information from additional sources (e.g. medical records, autopsy findings).

\section{Coordination of the consortium}

The Proof-ATHERO consortium is coordinated by the Clinical Epidemiology Team at the Medical University of Innsbruck, Austria. An outline of the processes involved in ProofATHERO coordination is provided in Fig. 2. Standardised data request forms are sent to eligible studies, inviting them to participate in the initiative. Upon receipt of study data, data cleaning 
and harmonisation are performed by a dedicated data management team using a range of tools for detecting inconsistencies and ambiguities in the data. Any queries arising during this process are clarified through direct correspondence with study investigators. Upon completion of the data management process, study data are stored in a central database at the coordinating centre. The data management system of the coordinating centre has been implemented in SAS 9.4. Proposals for analyses can be submitted by all members of the Proof-ATHERO study group (i.e. all named investigators of studies contributing data to Proof-ATHERO) via the consortium's webpage. Upon receipt, proposals are reviewed by a dedicated Proof-ATHERO steering committee, which then allocates resources at the coordinating centre according to resource availability and scientific priority of the project. For contractual reasons, data are stored and analysed exclusively at the Proof-ATHERO Coordinating and Statistics Centres (Medical University of Innsbruck and University of Cambridge). At each step from development of a statistical analysis plan, to the conduct of statistical analyses, and the creation of a manuscript draft, investigators of contributing studies and expert panels are contacted for feedback and comments, therefore making use of the broad and diverse community of experts in the field involved in the initiative.

\section{General approach to statistical analyses}

For each scientific project, statistical analyses will be performed according to a pre-specified analysis plan. Statistical analyses will follow established methods in the analysis of individualparticipant data [24-29]. Generally, the multi-level structure of data (e.g. multiple cohorts) will be taken into account by combining study-specific estimates using meta-analytical methods or by using mixed regression models with appropriate specification of random effects. Analyses will also involve assessments of between-studies heterogeneity. More details on specific analytical methods will be provided in publications resulting from each scientific project.

\section{Data protection and ethics considerations}

All studies contributing data to Proof-ATHERO have previously reported results and have obtained relevant local ethics approval and participants' consent. The data provided by each study remain entirely the property of the principal investigators of that study and are held in confidence by the Proof-ATHERO coordinating centre. To safeguard the identity of individuals at all stages of the analysis and to ensure compliance with data protection legislation and confidentiality guidelines, study data are transferred to the coordinating centre using encrypted 
connections. De-identified data are being stored securely in a central database at the coordinating centre, protected by firewalls and accessible only to authorised staff. Participants and collaborating studies have the right to withdraw from the Proof-ATHERO consortium at any time and without giving reasons.

\section{$\underline{\text { Characteristics of contributing studies }}$}

As of 24 January 2020, a total of 74 studies involving 106,846 participants are part of the ProofATHERO consortium. The designs of contributing studies and key study-level characteristics are shown in Table 2 . In summary, 21 studies recruited participants from the general population, 16 studies were conducted in patient populations with specific pre-existing diseases (e.g. with diabetes), and 37 studies were randomised controlled trials covering a range of different patient populations. The numbers of people enrolled in these three types of studies were 67,784, 22,677, and 16,385, respectively. Baseline years ranged from April 1980 to July 2014; the last follow-up was in June 2019. Mean age at baseline was 59 years (standard deviation: 10); 50\% of participants were female. Fig. 3 demonstrates the geographical location of contributing studies. Study locations were spread across four continents and are based in 25 countries and over 40 cities. The median duration of follow-up (i.e. the time from baseline to first event or end of follow-up) was 6.1 years (interquartile range: 2.7-10.4). Over a total of 830,619 person-years of follow-up, 17,270 incident CVD events and 13,270 deaths were recorded, corresponding to cumulative incidences of $2.1 \%$ and $1.6 \%$ per annum, respectively. As Proof-ATHERO evolves further, up-to-date information on contributing studies are being made available on the consortium's webpage at https://clinicalepi.i-med.ac.at/research/proofathero/.

\section{Initial set of hypotheses to be tested}

The large sample size and variety of data in Proof-ATHERO will enable us to test several hypotheses that are particularly power-hungry and could therefore not be addressed by previous studies. For instance, it is unclear whether cIMT progression could serve as a surrogate marker for hard cardiovascular outcomes in clinical trials [30-32]. Second, given conflicting results of prior individual studies [33-39], the comparative predictive value of cIMT measurements at different locations of the carotid artery remains to be determined in detail. Third, building on the initial insights of our recent literature-based meta-analysis [40], Proof-ATHERO will characterise in detail the association of cIMT with long-term risk of developing carotid plaque. 
In general, as a large-scale consortium of patient-level data, the high statistical power and consistent approach to statistical analysis and outcome definitions of Proof-ATHERO will help to address the aforementioned and other questions more reliably than previously possible.

\section{$\underline{\text { Strengths and limitations }}$}

Proof-ATHERO is a large consortium with a huge amount of data on atherosclerosis applying consistent approaches to data harmonisation and analysis. By inclusion of data from 25 countries and different clinical settings, the generalisability of findings will be of particular value. Our study also has several limitations. First, there were some differences between studies in how they assessed atherosclerosis measures and clinical outcomes. To address this issue, we collect meticulously a variety of study-specific characteristics, enabling us to quantify and better understand the impact of these differences in future analyses. Second, comprehensive data cleaning and harmonisation is a serious, often underestimated challenge. However, we managed to develop a sophisticated data management system that enables to transparently and effectively handle various datasets with different structures provided by the individual studies. Third, the current focus of available data lies on cIMT due to participation of multiple studies previously involved the PROG-IMT consortium [20]. Fourth, there exist several other markers for atherosclerosis, such as the assessment of endothelial function [41] with flow-mediated dilation or peripheral arterial tone, which have not been collected within Proof-ATHERO yet. Since the consortium is designed to continuously collect new data as they become available, coverage of other atherosclerosis markers will be expanded over time.

\section{Conclusion}

The Proof-ATHERO consortium is a multi-institutional collaborative project that is coordinated at the Medical University of Innsbruck, Austria. The consortium brings together large-scale data from prospective studies in the field of atherosclerosis. Proof-ATHERO combines data on CVD risk factors, repeat assessments of atherosclerosis, and clinical outcomes with cuttingedge data management and analytical tools. Building on these strengths, Proof-ATHERO will help to better characterise, understand, and predict the development of atherosclerosis and its clinical consequences. 


\section{Acknowledgement}

This manuscript was prepared using data of the Atherosclerosis Risk in Communities Study (ARIC), the Cardiovascular Health Study (CHS), the Jackson Heart Study (JHS), and the MultiEthnic Study of Atherosclerosis (MESA) obtained from the NHLBI Biologic Specimen and Data Repository Information Coordinating Center (BioLINCC) and does not necessarily reflect the opinions or views of ARIC, CHS, JHS, MESA, or NHLBI. An anonymised individualparticipant data dataset from CREED was kindly provided by Prof. Zoccali, Prof. Tripepi, and Prof. Mallamaci from the Institute of Biomedicine (CNR), Clinical Epidemiology and Physiopathology of Renal Diseases and Hypertension, Reggio Calabria, Italy on the basis on their 'public use' policy. We thank the CREED group for sharing their valuable data. We are grateful to the team members of the VASCage project (FFG COMET K-Project 843536) for their support.

\section{Disclosure Statement}

L. Tschiderer reports grants from the Dr.-Johannes-and-Hertha-Tuba Foundation during the conduct of the study and non-financial support from Sanofi outside the submitted work. L. Seekircher reports non-financial support from Sanofi outside the submitted work. G. Klingenschmid reports non-financial support from Sanofi and Pfizer outside the submitted work. N. Sattar reports personal fees from Amgen, AstraZeneca, Boehringer Ingelheim, EliLilly, Novo Nordisk, Sanofi, and Pfizer and grants from Boehringer Ingelheim outside the submitted work. S.E. Kjeldsen reports personal fees from Merck Darmstadt, MSD Whitehouse Station, Sanofi Paris, and Takeda Chicago outside the submitted work. S. Kiechl reports grants from Austrian Promotion Agency FFG outside the submitted work. G.D. Norata reports grants from Pfizer and Amgen and personal fees from Sanofi, Amgen, Alnylam, and Novartis outside the submitted work. P. Willeit reports grants from the Dr.-Johannes-and-Hertha-Tuba Foundation and the Austrian Science Fund FWF during the conduct of the study. Other authors have no conflicts of interest. 


\section{Funding Sources}

This work has been funded by the Austrian Science Fund (FWF) [P 32488] and the Dr.Johannes-and-Hertha-Tuba Foundation. Funders of individual studies contributing to the present analysis are listed on the Proof-ATHERO webpage (https://clinicalepi.imed.ac.at/research/proof-athero/studies/).

\section{Author Contributions}

L. Tschiderer, L. Seekircher, G. Klingenschmid, and P. Willeit are part of the coordinating centre and are responsible for data management and data analysis of the Proof-ATHERO consortium. L. Tschiderer and L. Seekircher drafted the manuscript, conducted the analyses, and interpreted the data. G. Klingenschmid interpreted the data. M. J. Sweeting provided supervision for statistical analyses. P. Willeit is responsible for the conception and design of the work, drafted the manuscript, conducted the analyses, and interpreted the data. All other authors were responsible for data acquisition. All authors revised the manuscript critically for important intellectual content approved the final version of the manuscript.

Collaborators committee: Maria V. Manzi ${ }^{1}$, Costantino Mancusi ${ }^{1}$, Helmuth Steinmetz ${ }^{2}$, Matthias Sitzer ${ }^{2,3}$, Mauro Amato ${ }^{4}$, Fabrizio Veglia ${ }^{4}$, Elena Tremoli ${ }^{4}$, Samuela Castelnuovo ${ }^{5}$, Dong Zhao ${ }^{6}$, Miao Wang ${ }^{6}$, Stela McLachlan ${ }^{7}$, Moo-Sik Lee ${ }^{8,9}$, Hyun-Woong Park ${ }^{9}$, Salim Yusuf $^{10,11}$, Diederick E. Grobbee ${ }^{12}$, Frank L. J. Visseren ${ }^{13}$, John J. P. Kastelein ${ }^{14}$, Wiek van Gilst $^{15}$, Folkert W. Asselbergs ${ }^{16}$, Muriel P. C. Grooteman ${ }^{17}$, Peter J. Blankestijn ${ }^{18}$, Ercan Ok ${ }^{19}$, Giuseppe Guglielmini ${ }^{20}$, Rino Migliacci ${ }^{21}$, Lena Bokemark ${ }^{22}$, Kazuo Kitagawa ${ }^{23}$, Michael Skilton $^{24}$, Lisa M. Jamieson ${ }^{25}$, Oscar Beloqui ${ }^{26}$, David Preiss ${ }^{27}$, Philip C. Calder ${ }^{28,29}$, Lokpal Bhatia $^{28,29}$, Pieter M. ter Wee ${ }^{17}$, Chrysostomos Dimitriadis ${ }^{30}$, Radovan Hojs ${ }^{31,32}$, Sebastjan Bevc $^{31,32}$, Peter Reiss ${ }^{33,34}$, Marit G. A. van Vonderen ${ }^{35}$, Ana R. Cunha ${ }^{36}$, Mayuko Amaha ${ }^{37}$, Tsukasa Nakamura ${ }^{37}$, Tatyana Balakhonova ${ }^{38}$, Maya Safarova ${ }^{39}$, Jesse Dawson ${ }^{40}$, Peter Higgins $^{40}$, Kristian Wachtell ${ }^{41}$, Sverre E. Kjeldsen ${ }^{41}$, Aleksandar Jovanovic ${ }^{42}$, Tatjana Lazarevic $^{43}$, Aleksandra Araszkiewicz ${ }^{44}$, Aleksandra Uruska ${ }^{44}$, Dariusz Naskręt ${ }^{44}$, Beat Frauchiger $^{45}$, Heiko Uthoff ${ }^{46}$, Kazuomi Kario ${ }^{47}$, Satoshi Hoshide ${ }^{47}$, Erik Stroes ${ }^{14}$, Edith Beishuizen $^{48}$, Tadao Akizawa ${ }^{49}$, Thapat Wannarong ${ }^{50,51}$, Sophia Zoungas ${ }^{52}$, John McNeil ${ }^{52}$, Alfonsa Friera ${ }^{53}$, Carmen Suarez ${ }^{54}$, Femke Rutters ${ }^{55}$, Petra Elders ${ }^{56}$, Coen D. A. Stehouwer ${ }^{57}$, 
Moise Desvarieux ${ }^{58,59}$, Pierre Ducimetiere ${ }^{60}$, Matthieu Plichart ${ }^{61,62}$, Hertzel C. Gerstein ${ }^{10,11}$, Ari Voutilainen $^{63}$, Jussi Kauhanen ${ }^{63}$, Liliana Grigore ${ }^{64}$, Giuseppe D. Norata ${ }^{64,65}$, Ta-Chen Su ${ }^{66}$, PeiChun Chen ${ }^{67}$, Hung-Ju Lin ${ }^{66}$, Holger Poppert ${ }^{68}$, Horst Bickel ${ }^{69}$, and M. Arfan Ikram ${ }^{70}$.

Affiliations of members of the collaborators committee: ${ }^{1}$ Department of Advanced Biochemical Sciences, Federico II University, Naples, Italy; ${ }^{2}$ Department of Neurology, Goethe University, Frankfurt am Main, Germany; ${ }^{3}$ Department of Neurology, Klinikum Herford, Herford, Germany; ${ }^{4}$ Centro Cardiologico Monzino IRCCS, Milan, Italy; ${ }^{5}$ Centro Dislipidemie, ASST Grande Ospedale Metropolitano Niguarda, Milan, Italy; ${ }^{6}$ Department of Epidemiology, Beijing Anzhen Hospital, Capital Medical University, Beijing, China; ${ }^{7}$ Usher Institute, University of Edinburgh, Edinburgh, UK; ${ }^{8}$ Department of Preventive Medicine, Konyang University, Daejeon, Korea; ${ }^{9}$ College of Medicine, Konyang University Hospital, Daejeon, Korea; ${ }^{10}$ Department of Medicine and Population Health Research Institute, McMaster University, Hamilton, Ontario, Canada; ${ }^{11}$ Hamilton General Hospital, Hamilton, Ontario, Canada; ${ }^{12}$ Julius Center for Health Sciences and Primary Care, University Medical Center Utrecht, Utrecht, the Netherlands; ${ }^{13}$ Department of Vascular Medicine, University Medical Center Utrecht, Utrecht, the Netherlands; ${ }^{14}$ Department of Vascular Medicine, Academic Medical Centre, University of Amsterdam, Amsterdam, the Netherlands; ${ }^{15}$ Department of Experimental Cardiology, University Medical Center Groningen, Groningen, the Netherlands; ${ }^{16}$ Department of Cardiology, University Medical Center Utrecht, Utrecht, the Netherlands; ${ }^{17}$ Department of Nephrology, Amsterdam UMC, Amsterdam, the Netherlands; ${ }^{18}$ Department of Nephrology, University Medical Center Utrecht, Utrecht, the Netherlands; ${ }^{19}$ Nephrology Department, Ege University School of Medicine, Bornova-Izmir, Turkey; ${ }^{20}$ Division of Internal and Cardiovascular Medicine, Department of Medicine, University of Perugia, Perugia, Italy; ${ }^{21}$ Division of Internal Medicine, Cortona Hospital, Cortona, Italy; ${ }^{22}$ Wallenberg Laboratory for Cardiovascular Research, University of Gothenburg, Gothenburg, Sweden; ${ }^{23}$ Department of Neurology, Tokyo Women's Medical University, Tokyo, Japan; ${ }^{24}$ Boden Institute of Obesity, Nutrition, Exercise and Eating Disorders, University of Sydney, Sydney, NSW 2006, Australia; ${ }^{25}$ Australian Research Centre for Population Oral Health, University of Adelaide, Adelaide, SA, Australia; ${ }^{26}$ Department of Internal Medicine, University Clinic of Navarra, Navarra, Spain; ${ }^{27}$ MRC Population Health Research Unit, Clinical Trial Service Unit, Nuffield Department of Population Health, University of Oxford, Oxford, UK; ${ }^{28}$ Faculty of Medicine, University of Southampton - Southampton General Hospital, Southampton, UK; ${ }^{29}$ Southampton NIHR Biomedical Research Centre, University Hospital Southampton - Southampton General 
Hospital, Southampton, UK; ${ }^{30}$ University Department of Nephrology, Hippokration General Hospital, Thessaloniki, Greece; ${ }^{31}$ Department of Nephrology, University Medical Centre Maribor, Maribor, Slovenia; ${ }^{32}$ Faculty of Medicine, University of Maribor, Maribor, Slovenia; ${ }^{33}$ Department of Global Health, Amsterdam UMC- Location AMC, Amsterdam, the Netherlands; ${ }^{34}$ Amsterdam Institute for Global Health and Development, University of Amsterdam, Amsterdam, the Netherlands; ${ }^{35}$ Department of Internal Medicine, Medical Center Leeuwarden, Leeuwarden, the Netherlands; ${ }^{36}$ Department of Clinical Medicine, State University of Rio de Janeiro, Rio de Janeiro, Brazil; ${ }^{37}$ Division of Nephrology, Shinmatsudo Central General Hospital, Chiba, Japan; ${ }^{38}$ Ultrasound Vascular Laboratory, National Medical Research Center of Cardiology, Moscow, Russia; ${ }^{39}$ Atherosclerosis Department, National Medical Research Center of Cardiology, Moscow, Russia; ${ }^{40}$ Institute of Cardiovascular and Medical Sciences, University of Glasgow, Glasgow, UK; ${ }^{41}$ Department of Cardiology, Oslo University Hospital, Oslo, Norway; ${ }^{42}$ Faculty of Medicine, University of Prishtina, PrishtinalKosovska Mitrovica, Serbia; ${ }^{43}$ Faculty of Medicine, University of Kragujevac, Kragujevac, Serbia; ${ }^{44}$ Department of Internal Medicine and Diabetology, Poznan University of Medical Sciences, Poznan, Poland; ${ }^{45}$ Department of Internal Medicine, Kantonsspital Frauenfeld, Frauenfeld, Switzerland; ${ }^{46}$ Department of Angiology, University Hospital Basel, Basel, Switzerland; ${ }^{47}$ Department of Medicine, Jichi Medical University School of Medicine, Tochigi, Japan; ${ }^{48}$ Department of Internal Medicine, HMC+ (Bronovo), the Hague, the Netherlands; ${ }^{49}$ Division of Nephrology, Department of Medicine, Showa University School of Medicine, Tokyo, Japan; ${ }^{50}$ Stroke Prevention \& Atherosclerosis Research Centre, Western University, London, Canada; ${ }^{51}$ Department of Internal Medicine, Faculty of Medicine, Siriraj Hospital, Mahidol University, Bangkok, Thailand; ${ }^{52}$ School of Public Health and Preventive Medicine, Monash University, Melbourne, Australia; ${ }^{53}$ Radiology Department, Universidad Autónoma de Madrid, Madrid, Spain; ${ }^{54}$ Internal Medicine Department, Universidad Autónoma de Madrid, Madrid, Spain; ${ }^{55}$ Department of Epidemiology \& Biostatistics, Amsterdam UMCLocation Vumc, Amsterdam, the Netherlands; ${ }^{56}$ Department of General Practice, Amsterdam UMC- Location Vumc, Amsterdam, the Netherlands; ${ }^{57}$ Department of Internal Medicine and Cardiovascular Research Institute Maastricht (CARIM), Maastricht University Medical Centre, Maastricht, the Netherlands; ${ }^{58}$ Department of Epidemiology, Mailman School of Public Health, Columbia University, New York, USA; ${ }^{59}$ METHODS Core, Centre de Recherche Epidémiologie et Statistique Paris Sorbonne Cité (CRESS), Institut National de la Santé et de la Recherche Médicale (INSERM) UMR 1153, Paris, France; ${ }^{60}$ Faculty of Medicine, University Paris Descartes, Paris, France; ${ }^{61}$ Paris Cardiovascular Research Centre (PARCC), University 
Paris Descartes, Paris, France; ${ }^{62}$ Assistance Publique, Hôpitaux de Paris, Hôpital Broca, Paris, France; ${ }^{63}$ Institute of Public Health and Clinical Nutrition, University of Eastern Finland, Kuopio Campus, Kuopio, Finland; ${ }^{64}$ SISA Center for the Study of Atherosclerosis, Bassini Hospital, Cinisello Balsamo, Italy; ${ }^{65}$ Department of Pharmacological and Biomolecular Sciences, University of Milan, Milan, Italy; ${ }^{66}$ Department of Internal Medicine, National Taiwan University Hospital, Taipei, Taiwan; ${ }^{67}$ Clinical Informatics \& Medical Statistics Research Center, Chang Gung University, Taoyuan, Taiwan; ${ }^{68}$ Department of Neurology, Technische Universität München, Munich, Germany; ${ }^{69}$ Department of Psychiatry and Psychotherapy, Technische Universität München, Munich, Germany; ${ }^{70}$ Department of Epidemiology, Erasmus University Medical Center, Rotterdam, the Netherlands 


\section{References}

1 GBD 2017 Causes of Death Collaborators: Global, regional, and national age-sex-specific mortality for 282 causes of death in 195 countries and territories, 1980-2017: a systematic analysis for the Global Burden of Disease Study 2017. Lancet 2018;392:1736-1788.

2 Enos WF, Holmes RH, Beyer J: Coronary disease among United States soldiers killed in action in Korea; preliminary report. J Am Med Assoc 1953;152:1090-1093.

3 McNamara JJ, Molot MA, Stremple JF, Cutting RT: Coronary artery disease in combat casualties in Vietnam. JAMA 1971;216:1185-1187.

4 Libby P, Ridker PM, Hansson GK: Progress and challenges in translating the biology of atherosclerosis. Nature 2011;473:317-325.

5 Ebrahim S, Papacosta O, Whincup P, Wannamethee G, Walker M, Nicolaides AN, et al.: Carotid plaque, intima media thickness, cardiovascular risk factors, and prevalent cardiovascular disease in men and women: the British Regional Heart Study. Stroke 1999;30:841-850.

6 Müller-Scholden L, Kirchhof J, Morbach C, Breunig M, Meijer R, Rücker V, et al.: Segment-specific association of carotid-intima-media thickness with cardiovascular risk factors - findings from the STAAB cohort study. BMC Cardiovasc Disord 2019;19:84.

7 Pignoli P, Tremoli E, Poli A, Oreste P, Paoletti R: Intimal plus medial thickness of the arterial wall: A direct measurement with ultrasound imaging. Circulation 1986;74:13991406.

8 Lorenz MW, Polak JF, Kavousi M, Mathiesen EB, Völzke H, Tuomainen T-P, et al.: Carotid intima-media thickness progression to predict cardiovascular events in the general population (the PROG-IMT collaborative project): A meta-analysis of individual participant data. Lancet 2012;379:2053-2062.

9 Den Ruijter HM, Peters SAE, Anderson TJ, Britton AR, Dekker JM, Eijkemans MJ, et al.: Common carotid intima-media thickness measurements in cardiovascular risk prediction: A meta-analysis. JAMA 2012;308:796-803.

10 Kiechl S, Willeit J: The natural course of atherosclerosis. Part I: Incidence and progression. Arterioscler Thromb Vasc Biol 1999;19:1484-1490.

11 Kiechl S, Willeit J: The natural course of atherosclerosis. Part II: Vascular remodeling. Bruneck Study Group. Arterioscler Thromb Vasc Biol 1999;19:1491-1498. 
12 Fowkes FGR, Murray GD, Butcher I, Heald CL, Lee RJ, Chambless LE, et al.: Ankle brachial index combined with Framingham Risk Score to predict cardiovascular events and mortality: A meta-analysis. JAMA 2008;300:197-208.

13 Ben-Shlomo Y, Spears M, Boustred C, May M, Anderson SG, Benjamin EJ, et al.: Aortic pulse wave velocity improves cardiovascular event prediction: An individual participant meta-analysis of prospective observational data from 17,635 subjects. J Am Coll Cardiol 2014;63:636-646.

14 Kramer CK, Zinman B, Gross JL, Canani LH, Rodrigues TC, Azevedo MJ, et al.: Coronary artery calcium score prediction of all cause mortality and cardiovascular events in people with type 2 diabetes: Systematic review and meta-analysis. BMJ 2013;346:f1654.

15 Kavousi M, Desai CS, Ayers C, Blumenthal RS, Budoff MJ, Mahabadi A-A, et al.: Prevalence and Prognostic Implications of Coronary Artery Calcification in Low-Risk Women: A Meta-analysis. JAMA 2016;316:2126-2134.

16 Chaikriangkrai K, Jhun HY, Palamaner Subash Shantha G, Bin Abdulhak A, Sigurdsson G, Nabi F, et al.: Coronary artery calcium score as a predictor for incident stroke: Systematic review and meta-analysis. Int J Cardiol 2017;236:473-477.

17 Vlachopoulos C, Xaplanteris P, Aboyans V, Brodmann M, Cífková R, Cosentino F, et al.: The role of vascular biomarkers for primary and secondary prevention. A position paper from the European Society of Cardiology Working Group on peripheral circulation: Endorsed by the Association for Research into Arterial Structure and Physiology (ARTERY) Society. Atherosclerosis 2015;241:507-532.

18 Rajzer MW, Wojciechowska W, Klocek M, Palka I, Brzozowska-Kiszka M, KaweckaJaszcz K: Comparison of aortic pulse wave velocity measured by three techniques: Complior, SphygmoCor and Arteriograph. J Hypertens 2008;26:2001-2007.

19 Knuuti J, Wijns W, Saraste A, Capodanno D, Barbato E, Funck-Brentano C, et al.: 2019 ESC Guidelines for the diagnosis and management of chronic coronary syndromes. Eur Heart J 2019;100:106.

20 Lorenz MW, Bickel H, Bots ML, Breteler MMB, Catapano AL, Desvarieux M, et al.: Individual progression of carotid intima media thickness as a surrogate for vascular risk (PROG-IMT): Rationale and design of a meta-analysis project. Am Heart J 2010;159:730736.e2.

21 Lorenz MW, Price JF, Robertson C, Bots ML, Polak JF, Poppert H, et al.: Carotid intimamedia thickness progression and risk of vascular events in people with diabetes: Results from the PROG-IMT collaboration. Diabetes Care 2015;38:1921-1929. 
22 Lorenz MW, Gao L, Ziegelbauer K, Norata GD, Empana JP, Schmidtmann I, et al.: Predictive value for cardiovascular events of common carotid intima media thickness and its rate of change in individuals at high cardiovascular risk - Results from the PROG-IMT collaboration. PLoS ONE 2018;13:e0191172.

23 Gray-Weale AC, Graham JC, Burnett JR, Byrne K, Lusby RJ: Carotid artery atheroma: comparison of preoperative B-mode ultrasound appearance with carotid endarterectomy specimen pathology. J Cardiovasc Surg (Torino) 1988;29:676-681.

24 Thompson S, Kaptoge S, White I, Wood A, Perry P, Danesh J: Statistical methods for the time-to-event analysis of individual participant data from multiple epidemiological studies. Int J Epidemiol 2010;39:1345-1359.

25 Jackson D, White I, Kostis JB, Wilson AC, Folsom AR, Wu K, et al.: Systematically missing confounders in individual participant data meta-analysis of observational cohort studies. Stat Med 2009;28:1218-1237.

26 Pennells L, Kaptoge S, White IR, Thompson SG, Wood AM: Assessing risk prediction models using individual participant data from multiple studies. Am J Epidemiol 2014;179:621-632.

27 Sanderson J, Thompson SG, White IR, Aspelund T, Pennells L: Derivation and assessment of risk prediction models using case-cohort data. BMC Med Res Methodol 2013;13:113.

28 White IR: Multivariate random-effects meta-analysis. Stata Journal 2009;9:40-56. http://www.stata-journal.com/article.html?article=st0156.

29 Riley RD, Price MJ, Jackson D, Wardle M, Gueyffier F, Wang J, et al.: Multivariate metaanalysis using individual participant data. Res Synth Methods 2015;6:157-174.

30 Espeland MA, O'Leary DH, Terry JG, Morgan T, Evans G, Mudra H: Carotid intimalmedia thickness as a surrogate for cardiovascular disease events in trials of HMG-CoA reductase inhibitors. Curr Control Trials Cardiovasc Med 2005;6:3.

31 Goldberger ZD, Valle JA, Dandekar VK, Chan PS, Ko DT, Nallamothu BK: Are changes in carotid intima-media thickness related to risk of nonfatal myocardial infarction? A critical review and meta-regression analysis. Am Heart J 2010;160:701-714.

32 Costanzo P, Perrone-Filardi P, Vassallo E, Paolillo S, Cesarano P, Brevetti G, et al.: Does carotid intima-media thickness regression predict reduction of cardiovascular events? A meta-analysis of 41 randomized trials. J Am Coll Cardiol 2010;56:2006-2020.

33 O'Leary DH, Polak JF, Kronmal RA, Manolio TA, Burke GL, Wolfson SK: Carotid-artery intima and media thickness as a risk factor for myocardial infarction and stroke in older 
adults. Cardiovascular Health Study Collaborative Research Group. N Engl J Med 1999;340:14-22.

34 Chambless LE, Folsom AR, Clegg LX, Sharrett AR, Shahar E, Nieto FJ, et al.: Carotid wall thickness is predictive of incident clinical stroke: the Atherosclerosis Risk in Communities (ARIC) study. Am. J. Epidemiol. 2000;151:478-487.

35 Lorenz MW, Kegler S von, Steinmetz H, Markus HS, Sitzer M: Carotid intima-media thickening indicates a higher vascular risk across a wide age range: prospective data from the Carotid Atherosclerosis Progression Study (CAPS). Stroke 2006;37:87-92.

36 Polak JF, Szklo M, O'Leary DH: Associations of Coronary Heart Disease with Common Carotid Artery Near and Far Wall Intima-Media Thickness: The Multi-Ethnic Study of Atherosclerosis. J Am Soc Echocardiogr 2015;28:1114-1121.

37 Lind L, Gigante B, Borne Y, Mälarstig A, Sundström J, Ärnlöv J, et al.: The plasma protein profile and cardiovascular risk differ between intima-media thickness of the common carotid artery and the bulb: A meta-analysis and a longitudinal evaluation. Atherosclerosis 2020;295:25-30.

38 Bots ML, Jong PTVM de, Hofman A, Grobbee DE: Left, Right, Near or Far Wall Common Carotid Intima-Media Thickness Measurements: Associations with Cardiovascular Disease and Lower Extremity Arterial Atherosclerosis. J Clin Epidemiol 1997;50:801-807.

39 Iglesias del Sol A, Bots ML, Grobbee DE, Hofman A, Witteman JCM: Carotid intimamedia thickness at different sites: relation to incident myocardial infarction; The Rotterdam Study. Eur Heart J 2002;23:934-940.

40 Tschiderer L, Klingenschmid G, Seekircher L, Willeit P: Carotid intima-media thickness predicts carotid plaque development: Meta-analysis of seven studies involving 9,341 participants. Eur J Clin Invest 2020:e13217.

41 Deanfield JE, Halcox JP, Rabelink TJ: Endothelial function and dysfunction: testing and clinical relevance. Circulation 2007;115:1285-1295. 


\section{Figure Legends}

Fig. 1. Measures for quantifying atherosclerosis.

Fig. 2. Data management and analysis workflow in the Proof-ATHERO consortium.

Fig. 3. Location of studies contributing data to the Proof-ATHERO consortium as of 24 January 2020. Full study names and references are provided in Table S1. 